\title{
THE EFFECT OF COVER CROP AND CROP ROTATION ON SOIL WATER STORAGE AND ON SORGHUM YIELD ${ }^{1}$
}

\author{
DEMÓSTENES MARCOS PEDROSA DE AZEVEDO², JUAN LANDIVAR ${ }^{3}$, \\ ROBSON MACEDO VIEIRA ${ }^{2}$ and DARYL MOSELEY ${ }^{4}$
}

\begin{abstract}
Crop rotation and cover crop can be important means for enhancing crop yield in rainfed areas such as the lower Coastal Bend Region of Texas, USA. A trial was conducted in 1995 as part of a long-term cropping experiment (7 years) to investigate the effect of oat (Avena sativa $\mathrm{L}$.) cover and rotation on soil water storage and yield of sorghum (Sorghum bicolor L.). The trial design was a RCB in a split-plot arrangement with four replicates. Rotation sequences were the main plots and oat cover crop the subplots. Cover crop reduced sorghum grain yield. This effect was attributed to a reduced concentration of available soil $\mathrm{N}$ and less soil water storage under this treatment. By delaying cover termination, the residue with a high $\mathrm{C} / \mathrm{N}$ acted as an $\mathrm{N}$ sink through competition and/or immobilization instead of an $\mathrm{N}$ source to sorghum plants. Crop rotation had a significantly positive effect on sorghum yield and this effect was attributed to a significantly larger amount of $\mathrm{N}$ concentration under these rotation sequences.
\end{abstract}

Index terms: rotation sequence, soybean, cotton.

\section{EFEITO DE CULTURA DE COBERTURA E DE ROTAÇÃO DE CULTURA NO ARMAZENAMENTO DE ÁGUA DO SOLO E NO RENDIMENTO DE SORGO}

\begin{abstract}
RESUMO - Rotação de cultura e cultura de cobertura constituem importantes meios para melhoria do rendimento de culturas em áreas de sequeiro como a região "Coastal Bend" do Estado do Texas. Um ensaio foi conduzido em 1995, como parte de um experimento de longa duração (7 anos), com o objetivo de investigar o efeito da aveia (Avena sativa L.) como cultura de cobertura, e da rotação de cultura, no armazenamento da água do solo e no rendimento do sorgo (Sorghum bicolor L.). O delineamento experimental adotado foi o de blocos ao acaso, em parcelas subdivididas, com quatro repetições. As rotações foram alocadas nas parcelas, e a cultura de cobertura, nas subparcelas. A cultura de cobertura reduziu o rendimento do sorgo. Este efeito foi atribuído à reduzida concentração de $\mathrm{N}$ disponível do solo. Por atraso no extermínio e incorporação da aveia, seu resíduo, com elevada relação $\mathrm{C} / \mathrm{N}$, atuou como dreno, pela imobilização, em lugar de ser fonte de $\mathrm{N}$ para o sorgo. As rotações sorgo/algodão e sorgo/soja proporcionaram efeito positivo no rendimento de sorgo. Este efeito foi atribuído à maior concentração de $\mathrm{N}$ nestas seqüências.
\end{abstract}

Termos para indexação: seqüência de rotação, soja, algodão.

\section{INTRODUCTION}

Available soil water and soil $\mathrm{N}$ are two factors of major importance in crop production. Cropping prac-

\footnotetext{
${ }^{1}$ Accepted for publication on June 4, 1998.

2 Agronomist, Ph.D., Embrapa-Centro Nacional de Pesquisa de Algodão (CNPA), Caixa Postal 174, CEP 58107-720 Campina Grande, PB, Brazil. E-mail: azevedo@cnpa.embrapa.br

${ }^{3}$ Agronomist, Ph.D., Texas A\&M University Agricultural Research \& Extension Center, Corpus Christi, TX, USA.

${ }^{4}$ Agronomist, M.Sc., Texas A\&M University Agricultural Research \& Extension Center, Corpus Christi, TX, USA.
}

tices, such as use of cover crop in rotation systems, used to improve soil moisture storage and accumulation of soil nitrate-N from decomposing organic matter would stabilize crop yields in rainfed areas such as the Lower Coastal Bend Region of Texas.

Cover crop can maintain or increase crop yield by reducing soil erosion, increasing soil organic matter, improving soil physical properties, reducing water runoff, reducing chemical inputs and maintaining or improving water quality (Holderbaum et al., 1990; Meissinger et al., 1991). Response to dry-matter accumalation and early 
season soil water storage by cover crop depend basically on three factors: weather, soil-holding capacity, and cover crop management (Wagger, 1987; Wagger \& Mengel, 1988). Under humid conditions, soil water depletion by cover crop is not a critical factor particularly on soil with high water-holding capacity. On soil with low water-holding capacity, dry weather conditions and erratic distribution of summer rainfall often fails to recharge profile soil moisture during the growing season. This condition would be more aggravated by the presence of cover crop prior to planting the summer crop. To minimize the effect of soil-water depletion by cover crops, early herbicide desiccation, animal grazing, or mechanical harvesting could be used (Campbell et al., 1984). The effectiveness of a cover crop in maintaining or improving soil water storage also depends on other factors such as: cover crop species, method of planting, stage of growth, length and growth period, method of extermination, leaving the crop as a mulch or green manure (Moschler et al., 1967; Nelson et al., 1977; Campbell et al., 1984).

Crop rotation practice has been well established in rainfed agriculture. Beneficial effects of rotation have been reported to include improvements in soil moisture (Benson, 1985; Roder et al., 1989), soil nutrients (Carter \& Hartwig, 1962; Peterson \& Vargel, 1989), soil structure (Barber, 1972; Dick \& Vandoren, 1985; Griffith et al., 1988), soil microbes (Williams \& Schmitthenner, 1962; Cock, 1984), weeds (Slife, 1976; Bhowmik \& Doll, 1982), insects (Benson, 1985), diseases (Dick \& Vandoren, 1985; Dabney et al., 1988; Edwards et al., 1988), and phytotoxic compounds and/or growth promoting substances originating from crop residue (Primavesi, 1988).

The objective of this research was to investigate the effect of cover crop and crop rotation on soil water storage and to grain yield of sorghum.

\section{MATERIAL AND METHODS}

This study was part of a long-term rotation sequence initiated in 1988 at the Texas A\&M Agricultural Research and Extension Center, at Corpus Christi, USA, to evaluate cropping systems suitable for the lower Coastal Bend
Region of Texas. The experimental site included three soil types: Victoria clay soil series (fine montmorillonitic, hyperthermic, Type Pelluster), Clareville complex (Fine, montmorillonitic, hyperthermic Pachic Argiustolls), and Orelia fine sandy loam (Fine loamy, mixed hyperthermic Typic Ochraqualfs).

The experimental design was a randomized complete block in a split plot arrangement with four replicates. Crop rotations consisted of continuous sorghum, sorghum/cotton, sorghum/soybean, continuous cotton, cotton/sorghum, cotton/soybeans, soybean/sorghum, and soybean/cotton and were the main plots; cover crop (cover and no cover) was the subtreatment. Cover crop consisted of seeding feed grade oats in late September 1994 at a rate of approximately $170 \mathrm{~kg} / \mathrm{ha}$ and receiving glyphosate ( ( N - phosphonomethy) glycine) and 2,4 D ((2,4 - diclorophenoxy) acetic acid) at a rate of $2,4 \mathrm{~L} / \mathrm{ha}$ on January 13, 1995. The desiccated cover was allowed to remain in the field to provide a surface mulch until spring planting preparation; then, it was incorporated to the soil. Plant tissue samples were taken from the cover crop prior to extermination for dry matter yield and tissue analysis.

Data presented in this paper were collected in the 1995 growing season using only sorghum rotation sequences. Each plot was $13.7 \mathrm{~m}$ wide and $61 \mathrm{~m}$ long.

Sorghum rotations received preplant broadcast applications of $34 \mathrm{~kg} / \mathrm{ha}$ of $\mathrm{N}$ (32-0-0). A starter fertilizer, 8-24-2 rate of $30 \mathrm{~L} / \mathrm{ha}$ was also applied in $10 \mathrm{~cm}$ band over the seed furrow at planting. Sorghum cultivar DEKALB 56 was sown in $96 \mathrm{~cm}$ rows on March 26, 1995, at a population of 110,000 plants/ha. Weeds were controlled with a pre-emergence application of atrazine at a recommended rate. Two cultivations on April 24 and May 17 were needed to control remanecent weeds.

Soil water contents were monitored throughout the season using a neutron probe (Campbell Pacific Model 503, CPN Crop., Martinex, CA). Access tubes were installed in two locations (North and South) of each subplot at a sorghum/cotton rotation of four replicates after plant emergence. The probe was calibrated at each site by obtaining $15 \mathrm{~cm}$ increment soil samples and determining the soil water content. The gravimetric data were converted to a volumetric basis by multiplying them by the soil bulk density. Probe readings were taken twice a month during the growing season. Readings were taken from surface to a depth of $90 \mathrm{~cm}$. Neutron probe readings were converted to volumetric soil water contents by use of a regression equation to determine the amount of water in the soil profile. Volumetric soil values for the experimental area ranged from approximately $42 \%$ at 
saturation to $34 \%$ at field capacity to $18 \%$ at permanent wilting point (Lawlor et al., 1992). Plant extractable water (PEW) was determined as the difference between the volumetric soil moisture (VSM) measured between dates when little or no rainfall occurred. This is a modified laboratory and field method used by Ratliff et al. (1983) and Ritchie (1981), cited by Lawlor et al. (1992) to determine potential extractable water. Soil water depletion (WD) was determined as the difference between total water content on April 28 and July 28. Evapotranspiration (ET) was calculated as the sum of soil water depletion (WD) in the whole profile and precipitation received during the same experimental period, i.e., ET=WD + rainfall. Water runoff was assumed negligible because the slope was $<2 \%$. Water use efficiency (WUE) was calculated as gain yield (Y) divided by ET, or WUE=Y/ET (Tanner \& Sinclair, 1983). Data were analyzed by the standard analysis of variance procedures.

\section{RESULTS AND DISCUSSION}

\section{Oat dry matter and nitrogen content}

Dry matter yield (DM) and $\mathrm{N}$ concentration constitute an estimation of the $\mathrm{N}$ content of the above ground portion of a cover crop. These values represent $\mathrm{N}$ uptake from residual soil inorganic $\mathrm{N}$, mineralized $\mathrm{N}$, and biologically fixed $\mathrm{N}$ in legume cover crop. Mean values of oat DM and $\mathrm{N}$ content harvested in January of 1995 are presented in Table 1. Oat yield ranged from $1,449 \mathrm{~kg} / \mathrm{ha}$ to $1,842 \mathrm{~kg} / \mathrm{ha}$. Oat yield was higher under rotation when compared with continuous sorghum. Sorghum/cotton was the crop sequence that improved most cover

TABLE 1. Dry matter yield and nitrogen content of oat leaves at harvest. Corpus Christi, TX, 1995.

\begin{tabular}{lcc}
\hline Rotation sequence & $\begin{array}{c}\text { Dry matter } \\
\text { yield }(\mathrm{kg} / \mathrm{ha})\end{array}$ & $\begin{array}{c}\text { Nitrogen } \\
\text { content }(\mathrm{g} / \mathrm{kg})^{1}\end{array}$ \\
\hline Sorghum/sorghum & $1,449.5$ & $19.1 \mathrm{~b}$ \\
Sorghum/cotton & $1,842.3$ & $23.6 \mathrm{a}$ \\
Sorgum/soybean & $1,686.5$ & $22.7 \mathrm{a}$ \\
Mean & $1,659.4$ & 21.8 \\
LSD $(0.05)$ & $\mathrm{NS}^{2}$ & 3.1 \\
CV $(\%)$ & 26.9 & 8.1 \\
\hline
\end{tabular}

${ }^{1}$ Mean values followed by the same letter are not significantly different at $\mathrm{P}<0.05$ as determined by LSD test.

2 Test $\mathrm{F}$ nonsignificant at $\mathrm{P}<0.05$. crop dry matter yield. For $\mathrm{N}$ content, the highest mean value was registered under sorghum/cotton rotation $(23.6 \mathrm{~g} / \mathrm{kg})$ which differed significantly $(\mathrm{P}<0.05)$ only from sorghum/sorghum sequence. This result was somehow expected because of the higher nutrient demand by continuous sorghum sequence.

\section{Soil organic matter and soil nitrogen}

Results of organic matter (OM) and total $\mathrm{N}$ determinations are presented in Table 2. ANOVA indicates that only the main effect of rotation and depth on $\mathrm{OM}$ were significant $(\mathrm{P}<0.05$ and $\mathrm{P}<0.01$, respectively). Higher $\mathrm{OM}$ means were registered under rotations which did not significantly differ from each other but differed from continuous sorghum. Analysis of variance for this variable indicates a significant rotation $\mathrm{x}$ cover crop interaction $(\mathrm{P}<0.05)$. Soil $\mathrm{N}$ content was generally higher under sorghum/cotton and sorghum/soybean.

Within cover crop regime, no significant difference was observed between sequence treatments. Within no cover regime, the largest $\mathrm{N}$ concentration was registered on sorghum/cotton, which did not differ from sorghum/soybean, but differed from continuous sorghum. Cover crop significantly reduced soil $\mathrm{N}$ concentration.

\section{Soil water content and crop water use}

Average air temperature and cumulative precipitation for a seven-day period during the growing season are presented in Table 3. Rainfall from March to August 1995 was $96 \%$ of the 15 -year average precipitation of $369 \mathrm{~mm}$. Average temperatures were adequate for optimum growth and development of sorghum. However, seasonal variations in the amount and distribution of rain occurred throughout the season. Severe soil moisture deficit was observed at the critical stage of plant development, i.e, from planting-to-bloom period ( 1 - 60 days after planting).

Conservation of soil moisture by nonleguminous cover crop and crop rotation during the summer growing season is known to be related to reduced water evaporation, reduced rainfall runoff, increasing 
TABLE 2. Effect of crop rotation and cover crop on soil organic matter and nitrogen. Corpus Christi, TX, 1995 .

\begin{tabular}{lccc}
\hline Rotation sequence & Cover crop & $\begin{array}{c}\text { Organic matter } \\
(\%)\end{array}$ & $\begin{array}{c}\text { Nitrogen } \\
(\mathrm{mg} / \mathrm{kg})\end{array}$ \\
\hline Sorghum/sorghum & Cover & 0.57 & $3.10 \mathrm{bc}$ \\
& No cover & 0.61 & $4.15 \mathrm{~b}$ \\
Sorghum/cotton & Cover & 0.64 & $4.00 \mathrm{bc}$ \\
& No cover & 0.71 & $7.55 \mathrm{a}$ \\
Sorghum/soybean & Cover & 0.68 & $2.45 \mathrm{c}$ \\
& No cover & 0.65 & $6.15 \mathrm{a}$ \\
Mean values & & & 3.63 \\
Sorghum/sorghum & & $0.59 \mathrm{~b}$ & 5.78 \\
Sorghum/cotton & & $0.67 \mathrm{a}$ & 4.30 \\
Sorghum/soybean & & $0.66 \mathrm{a}$ & $3.18 \mathrm{~b}$ \\
& Cover & 0.63 & $5.95 \mathrm{a}$ \\
Treatment effects & No cover & 0.66 & $\mathrm{NS}$ \\
Rotation sequence (R) & & & $* *$ \\
Cover crop (C) & & $*$ & $*$ \\
Depth (D) & & $\mathrm{NS}$ & $* *$ \\
R x C & & $\mathrm{NS}$ & $*$ \\
\hline
\end{tabular}

${ }^{1}$ Mean values followed by the same letter within a column are not significantly different at $\mathrm{P}<0.05$.

2 Teste $\mathrm{F}$ nonsignificant at $\mathrm{P}<0.05$.

*, ** Significant at $\mathrm{P}<0.05$ and $\mathrm{P}<0.01$ respectively.

TABLE 3. Weekly mean temperature and cumulative precipitation during the growing season. Corpus Christi, TX, 1995.

\begin{tabular}{|c|c|c|c|c|c|}
\hline \multirow[t]{2}{*}{ Month } & \multirow[t]{2}{*}{ Week $^{1}$} & \multirow[t]{2}{*}{ Day } & \multirow{2}{*}{$\begin{array}{c}\text { Temperature } \\
\left({ }^{\circ} \mathrm{C}\right)\end{array}$} & \multicolumn{2}{|c|}{ Precipitation } \\
\hline & & & & $\begin{array}{c}\text { Weekly } \\
(\mathrm{mm})\end{array}$ & $\begin{array}{r}\text { Sum } \\
(\mathrm{mm})\end{array}$ \\
\hline March & 1 & $1-7$ & 19.1 & 11 & 11 \\
\hline \multirow[t]{4}{*}{ April } & 2 & 8-14 & 19.4 & 3 & 14 \\
\hline & 3 & $15-21$ & 21.3 & 4 & 18 \\
\hline & 4 & $22-28$ & 24.1 & 0 & 18 \\
\hline & 5 & $29-35$ & 20.7 & 0 & 18 \\
\hline \multirow[t]{4}{*}{ May } & 6 & $36-42$ & 24.1 & 1 & 19 \\
\hline & 7 & $43-49$ & 25.5 & 5 & 24 \\
\hline & 8 & $50-56$ & 27.8 & 8 & 32 \\
\hline & 9 & $57-63$ & 24.6 & 0 & 32 \\
\hline \multirow[t]{5}{*}{ June } & 10 & $64-70$ & 26.3 & 66 & 98 \\
\hline & 11 & $71-77$ & 27.5 & 1 & 99 \\
\hline & 12 & $78-84$ & 24.9 & 32 & 131 \\
\hline & 13 & $85-91$ & 25.3 & 14 & 145 \\
\hline & 14 & $92-98$ & 26.9 & 10 & 155 \\
\hline \multirow[t]{4}{*}{ July } & 15 & 99-105 & 27.9 & 3 & 158 \\
\hline & 16 & $106-112$ & 27.8 & 0 & 158 \\
\hline & 17 & $113-119$ & 28.2 & 47 & 205 \\
\hline & 18 & $120-126$ & 29.9 & 0 & 205 \\
\hline \multirow[t]{2}{*}{ August } & 19 & $127-133$ & 28.8 & 36 & 241 \\
\hline & 20 & $134-140$ & 29.1 & 1 & 242 \\
\hline
\end{tabular}

${ }^{1}$ Beginning March 24 and ending August 8, 1995. 
soil $\mathrm{OM}$ and water infiltration rate. For the data collected in 1995, analysis of variance of soil moisture content (sorghum/cotton rotation) indicates that only the main effect of cover crop (May 9, May 23, and July 5) and depth at all readings were significant (Tables 4 e 5). Cover crop did not show any consistent beneficial effect on soil water storage at any depth throughout the growing season. Lower soil water content under cover crop was observed on March 28, May 23 and June 6, while

TABLE 4. Soil moisture content of sorghum/cotton rotation as influenced by cover crop (C) and depth (D) (March 28 - June 6, 1995). Corpus Christi, TX, 1995.

\begin{tabular}{|c|c|c|c|c|c|c|c|c|}
\hline \multirow{3}{*}{$\begin{array}{c}\text { Depth } \\
(\mathrm{cm})\end{array}$} & \multicolumn{2}{|c|}{ March 28} & \multicolumn{2}{|c|}{ May 9} & \multicolumn{2}{|c|}{ May 23} & \multicolumn{2}{|c|}{ June 6} \\
\hline & Cover & No cover & Cover & No cover & Cover & No cover & Cover & No cover \\
\hline & \multicolumn{8}{|c|}{ - } \\
\hline $0-15$ & 15.80 & 13.45 & 15.21 & 16.76 & - & - & - & - \\
\hline $15-30$ & 26.64 & 27.35 & 28.71 & 24.34 & 20.40 & 21.39 & 25.99 & 26.16 \\
\hline $30-45$ & 27.26 & 27.99 & 30.08 & 26.26 & 21.98 & 23.10 & 25.64 & 25.96 \\
\hline $45-60$ & 26.83 & 27.91 & 29.81 & 27.07 & 22.86 & 24.55 & 25.61 & 25.33 \\
\hline $60-75$ & 25.57 & 27.45 & 28.67 & 26.86 & 23.95 & 26.62 & 24.10 & 24.78 \\
\hline $75-90$ & 25.22 & 27.09 & 28.16 & 26.76 & 24.59 & 28.31 & 24.77 & 25.39 \\
\hline Mean & 24.56 & 25.29 & 26.77 & 24.67 & 22.75 & 24.79 & 25.22 & 25.25 \\
\hline \multicolumn{9}{|c|}{ Treatment effect } \\
\hline $\mathrm{C}$ & \multicolumn{2}{|c|}{$\mathrm{NS}^{1}$} & \multicolumn{2}{|c|}{$* *$} & \multicolumn{2}{|c|}{$*$} & \multicolumn{2}{|c|}{ NS } \\
\hline $\mathrm{D}$ & \multicolumn{2}{|c|}{$* *$} & \multicolumn{2}{|c|}{$* *$} & \multicolumn{2}{|c|}{$*$} & \multicolumn{2}{|c|}{ NS } \\
\hline $\mathrm{CxD}$ & \multicolumn{2}{|c|}{ NS } & \multicolumn{2}{|c|}{ NS } & \multicolumn{2}{|c|}{ NS } & \multicolumn{2}{|c|}{ NS } \\
\hline
\end{tabular}

1 Test $\mathrm{F}$ nonsignificant at $\mathrm{P}<0.01$.

*, ** Significant at $\mathrm{P}<0.05$ and $\mathrm{P}<0.01$ respectively.

TABLE 5. Soil moisture content of sorghum/cotton rotation as influenced by cover crop (C) and depth (D) (June 20 - July 28). Corpus Christi, TX, 1995.

\begin{tabular}{|c|c|c|c|c|c|c|c|c|}
\hline \multirow{3}{*}{$\begin{array}{l}\text { Depth } \\
(\mathrm{cm})\end{array}$} & \multicolumn{2}{|c|}{ Jun 20} & \multicolumn{2}{|c|}{ July 5} & \multicolumn{2}{|c|}{ July 18} & \multicolumn{2}{|c|}{ July 28} \\
\hline & Cover & No cover & Cover & No cover & Cover & No cover & Cover & No cover \\
\hline & \multicolumn{8}{|c|}{ - } \\
\hline $0-15$ & 36.41 & 36.11 & - & - & - & - & - & - \\
\hline $15-30$ & 24.62 & 24.56 & 19.34 & 18.32 & 17.62 & 17.33 & 16.50 & 16.16 \\
\hline $30-45$ & 24.41 & 24.64 & 20.83 & 19.67 & 18.90 & 18.53 & 17.33 & 17.12 \\
\hline $45-60$ & 24.62 & 24.14 & 21.82 & 20.18 & 19.69 & 19.08 & 18.12 & 17.55 \\
\hline $60-75$ & 23.91 & 23.91 & 22.43 & 20.86 & 20.37 & 19.74 & 18.66 & 17.74 \\
\hline $75-90$ & 24.61 & 24.68 & 24.18 & 22.40 & 22.43 & 21.55 & 21.32 & 19.76 \\
\hline Mean & 26.43 & 26.34 & 21.72 & 20.29 & 19.80 & 19.25 & 18.39 & 17.67 \\
\hline \multicolumn{9}{|c|}{ Treatment effect } \\
\hline $\mathrm{C}$ & \multicolumn{2}{|c|}{$\mathrm{NS}^{1}$} & \multicolumn{2}{|c|}{ ** } & \multicolumn{2}{|c|}{ NS } & \multicolumn{2}{|c|}{ NS } \\
\hline $\mathrm{D}$ & \multicolumn{2}{|c|}{$* *$} & \multicolumn{2}{|c|}{$* *$} & \multicolumn{2}{|c|}{ ** } & \multicolumn{2}{|c|}{$* *$} \\
\hline $\mathrm{CxD}$ & \multicolumn{2}{|c|}{ NS } & \multicolumn{2}{|c|}{ NS } & \multicolumn{2}{|c|}{ NS } & \multicolumn{2}{|c|}{ NS } \\
\hline
\end{tabular}

1 Test $\mathrm{F}$ nonsignificant at $\mathrm{P}<0.01$.

** Significant at $\mathrm{P}<0.01$. 
higher means were registrered on May 9, June 20, July 18 and July 28 at basically all profiles. Significant difference due to cover crop (main effect); however, it was observed only on May 9 and July 5. On May 23, no cover was significantly higher than cover crop soil water content mean. An analysis of the soil moisture content on the whole profile shows that cover crop did not contribute to any consistent beneficial effect on soil water storage throughout the summer season (Table 4, 5 and 6). Percent difference at the whole profile varied from -8.23 to 7.05. Values of PEW for periods with little or no rainfall showed significant effect of cover crop on this variable over the whole growing season, except for July 18 to July 28. Percent difference was generally negative, meaning that PEW value was higher under no cover regime (Table 7). Water depletion, evapotranspiration, water use efficiency, and grain sorghum yield were significantly lower under cover than under no cover crop residue (Table 8).

\section{Grain yield}

Yield and significance of $\mathrm{F}$ values determined from analysis of variance are shown in Table 9. Grain yield ranged from 2,892 kg/ha to $5,551.49 \mathrm{~kg} / \mathrm{ha}$. ANOVA indicates that the main effect of rotation, cover, and the interaction rotation $\mathrm{x}$ cover were

TABLE 6. Mean profile soil water content on sorghum/cotton rotation as influenced by cover crop. Corpus Christi, TX, 1995 .

\begin{tabular}{|c|c|c|c|}
\hline \multirow[t]{2}{*}{ Date } & \multicolumn{2}{|c|}{ Soil moisture content } & \multirow{2}{*}{$\begin{array}{c}\text { Percent } \\
\text { difference }^{2}\end{array}$} \\
\hline & \multicolumn{2}{|c|}{------(m $\left(\mathrm{m}^{3} / \mathrm{m}^{3} \times 100\right)$} & \\
\hline $4 / 28$ & 24.56 & 25.29 & -2.89 \\
\hline $5 / 09$ & 26.77 & $24.67 *$ & 8.51 \\
\hline $5 / 23$ & 22.75 & $24.79 *$ & -8.23 \\
\hline $6 / 06$ & 25.22 & 25.52 & -1.18 \\
\hline $6 / 20$ & 26.43 & 26.34 & 0.34 \\
\hline $7 / 05$ & 21.72 & $20.29 *$ & 7.05 \\
\hline $7 / 18$ & 19.80 & 19.25 & 2.86 \\
\hline $7 / 28$ & 18.39 & 17.67 & 4.07 \\
\hline
\end{tabular}

${ }^{1}$ Means followed by an asterisk are significantly different between cover treatments $(\mathrm{P}<0.05)$

${ }^{2}$ Calculated as $(\mathrm{C} / \mathrm{NC}-1.00) \times 100$. significant. Sorghum yield was consistently and significantly higher under rotation than under the continuous sorghum system. Sorghum/cotton produced $962 \mathrm{~kg} / \mathrm{ha}$ of grain more than sorghum in continuous sequence, while sorghum/soybean produced the increment of $1,505 \mathrm{~kg} / \mathrm{ha}$ of grain above sorghum/sorghum rotation which represents an increase of 48 percent. Within cover crop regime, the highest yield was registered on sorghum/cotton sequence which significantly differed from continuous sorghum. Within no cover treatment, sorghum/soybean was the rotation sequence which presented the largest yield $(5,551 \mathrm{~kg} / \mathrm{ha})$ differing from sorghum/cotton and sorghum/sorghum. Cover crop consistently reduced sorghum yield. Significant difference, however, was observed only on sorghum/soybean sequence (interaction effect). The positive effect of sorghum/soybean rotation on sorghum yield supports Crookston et al. (1988) findings. In their trial, corn yielded significantly better when rotated with soybean or follow.

The absence of cover crop had a beneficial effect on sorghum yield in this trial which could be attributed to a reduced amount of available soil $\mathrm{N}$ and less soil water storage under this treatment when compared to the no cover cropping system. On the other hand, the beneficial effect of crop rotation seems to be correlated to the soil $\mathrm{N}$ concentration, particularly under sorghum/soybean sequence.

TABLE 7. Mean profile plant extractable water influenced by cover crop on sorghum/cotton rotation. Corpus Christi, TX, 1995'.

\begin{tabular}{|c|c|c|c|}
\hline \multirow[t]{2}{*}{ Date } & \multicolumn{2}{|c|}{ Plant extractable water } & \multirow{2}{*}{$\begin{array}{c}\text { Percent } \\
\text { difference }^{2}\end{array}$} \\
\hline & \multicolumn{2}{|c|}{----- $\left(\mathrm{m}^{3} / \mathrm{m}^{3} \times 100\right)$} & \\
\hline $4 / 28-7 / 18$ & 6.51 & $8.31 *$ & -21.66 \\
\hline $4 / 28-7 / 28$ & 7.93 & $9.89 *$ & -19.82 \\
\hline $5 / 23-7 / 18$ & 3.02 & $5.83 *$ & -48.20 \\
\hline $5 / 23-7 / 28$ & 4.36 & $7.17^{*}$ & -39.19 \\
\hline $7 / 05-7 / 28$ & 3.34 & $2.62 *$ & 27.48 \\
\hline $7 / 18-7 / 28$ & 1.42 & 1.60 & -11.25 \\
\hline
\end{tabular}

${ }^{1}$ Means followed by an asterisk are significantly different between cover treatments $(\mathrm{P}<0.05)$.

${ }^{2}$ Calculated as $(\mathrm{C} / \mathrm{NC}-1.00) \times 100$ 
TABLE 8. Sorghum grain yield (Y), water depletion (WD), evapotranspiration (ET), and water use efficiency (WUE) as affected by cover crop on sorghum/cotton sequence. Corpus Christi, TX, 1995'.

\begin{tabular}{lcccc}
\hline Cover regime & $\mathrm{Y}$ & $\mathrm{WD}^{2}$ & $\mathrm{ET}^{3}$ & $\mathrm{WUE}^{4}$ \\
& $(\mathrm{Mg} / \mathrm{ha})$ & $--------(\mathrm{mm})$ & ------- & $(\mathrm{kg} / \mathrm{ha} / \mathrm{mm})$ \\
\hline Cover & $3,469.13 \mathrm{~b}$ & $7.93 \mathrm{~b}$ & $212.93 \mathrm{~b}$ & $16.29 \mathrm{~b}$ \\
No cover & $4,451.22 \mathrm{a}$ & $9.89 \mathrm{a}$ & $214.89 \mathrm{a}$ & $20.71 \mathrm{a}$ \\
F test & $* *$ & $* *$ & $* *$ & $* *$ \\
\hline
\end{tabular}

${ }^{1}$ Means followed with the same letter in a column are not significantly different.

2 Calculated as initial soil water content minus final soil water content.

${ }^{3}$ Calculated as WD plus precipitation.

${ }^{4}$ Calculated at $\mathrm{Y} / \mathrm{ET}$.

TABLE 9. Sorghum grain yield as affected by rotation and cover crop. Corpus Chisti, TX, 1995'.

\begin{tabular}{lcc}
\hline Rotation sequence & Cover crop & $\begin{array}{c}\text { Yield } \\
(\mathrm{kg} / \mathrm{ha})\end{array}$ \\
\hline Sorghum/sorghum & Cover & $2,892.83 \mathrm{~d}$ \\
No cover & $3,382.33 \mathrm{~cd}$ \\
Sorghum/cotton & Cover & $3,780.22 \mathrm{bc}$ \\
& No cover & $4,419.86 \mathrm{bc}$ \\
Sorghum/soybean & Cover & $3,734.33 \mathrm{bc}$ \\
& No cover & $5,551.49 \mathrm{a}$
\end{tabular}

Mean values

Sorghum/sorghum

$3,137.58 b$

Sorghum/cotton

$4,100.04 \mathrm{a}$

Sorghum/soybean

$4,642.91 \mathrm{a}$

Treatment effects

\begin{tabular}{lll} 
& Cover & $3,469.13 \mathrm{~b}$ \\
& No cover & $4,451.22 \mathrm{a}$ \\
Rotation sequence (RS) & & $*$ \\
Cover crop (C) & & $* *$ \\
R x C & $*$ \\
\hline
\end{tabular}

${ }^{1}$ Mean values followed by the same letter with a rotation sequence are not significantly different by LSD teste at $\mathrm{P}<0.05$.

*** Significant at $\mathrm{P}<0.05$ and $\mathrm{P}<0.01$ respectively.

\section{CONCLUSIONS}

1. Cover crop does not show any beneficial effect on soil water storage and on sorghum yield.
2. Crop rotation has a positive effect on sorghum yield.

\section{REFERENCES}

BARBER, S.A. Relation of weather to the influence of hay crops on subsequent corn yields on a Chalmers silt loam. Agronomy Journal, v.64, n.1, p.8-10, 1972.

BENSON, G.O. Why the reduced yields when corn follows corn and possible management responses. In: PROCEEDING CORN AND SORGHUM RESEARCH, 1985, Chicago. Conference... Washington: Am. Seed Trade Assoc., 1985. p.971-972

BHOWMIK, P.C.; DOLL, J.D. Corn and soybean response to allelopathic effects of weed and crop residues. Agronomy Journal, v.74, n.4, p.601-606, 1982.

CAMPBELL, R.B.; KARLEN, D.L.; SOJKA, R.E. Conservation tillage for maize production in the U.S. Southeastern Coastal Plain. Soil Tillage Research, v.4, p.511-529, 1984.

CARTER, J.L.; HARTWIG, E.E. The management of soybeans. Advance Agronomy, v.14, p.359-412, 1962.

COCK, R.J. Root health: importance and relationship to farming practices. In: BEZDICEK, D.F. (Ed.) Organic farming. Madison: American Society Agronomy, 1984.p.111-127. (ASA. Special Publisher, 46).

CROOKSTON, R.K.; KURLE, J.E.; LUESCHEN, W.E. Relative ability of soybean, fallow, and triacontanol to alleviate yield reduction associate with growing corn continuously. Crop Science, v. 28, p.145-147, 1988.

DABNEY, S.M.; McGAWLEY, E.C.; BOETHEL, D.J.; BERGER, D.A. Short-term crop rotation systems for soybean production. Agronomy Journal, v.80, n.2, p.197-204, 1988.

DICK, W.A.; VANDOREN, J.D.M. Continuous tillage and rotation combinations effects on corn, soybean, and oat yields. Agronomy Journal, v.77, n.3, p.459-465, 1985.

EDWARDS, J.H.; THURLOW, D.L.; EASON, J.T. Influence of tillage and crop rotation on yields of 
corn, soybean, and wheat. Agronomy Journal, v.80, n.1, p.76-80, 1988.

GRIFFITH, D.R.; KLADIVKO, E.J.; MANNERING, J.V.; WEST, T.D.; PARSONS, S.D. Longterm tillage and rotation effects on corn growth and yield on high and low organic matter, poorly drained soils. Agronomy Journal, v.80, n.4, p.599-605, 1988.

HOLDERBAUM, J.F.; DECKER, A.M.; MEISINGER, J.J.; MULFORED, F.R.; VOUGH, L.R. Fall-seeded legume cover crops for no-tillage corn in the humid East. Agronomy Journal, v.82, n.1, p.117-124, 1990.

LAWLOR, D.J.; LANDIVAR, J.A.; CRESHAW, C.; VASEK, J. Soil water storage and productivity of cotton in conventional vs. reduced tillage systems. In: BELTWIDE COTTON PHYSIOLOGY CONFERENCE, 1991, Memphis, TN. Proceedings... Memphis: National Cotton Council of America, 1992. p.1046-1047.

MEISSINGER, J.J.; HARGROVE, W.L.; MIKKELSON, R.L.; WILLIAMS, J.R.; BENSON, J.W. Effects of cover crops on ground water quality. In: HARGROVE, W.L. (Ed.). Cover crops for clean water. Ankeny: Soil and Water Conserv. Soc., 1991. p.121-130.

MOSCHLER, W.W.; SHEAR, G.M.; HALLOCK, D.L.; SEARS, R.D.; JONES, G.D. Winter cover crops for sod-planted corn: Their selection and management. Agronomy Journal, v.59, p.547-551, 1967.

NELSON, L.R.; GALLAHER, R.N.; BRUCE, R.R.; HOLMES, M.R. Production of corn and sorghum grain in double cropping systems. Agronomy Journal, v.69, n.1, p.41-45, 1977.

PETERSON, T.A.; VARGEL, G.E. Crop yield as affected by rotation and nitrogen rate. I. Soybean. Agronomy Journal, v.81, n.57, p.727-731, 1989.

PRIMAVESI, A. Manejo ecológico do solo. 9.ed. São Paulo: Nobel, 1988. 549p.
RATLIFF, L.F.; RITCHIE, J.J.; CASSEL, D.K. Field measured limits on soil water availability as related to laboratory-measured properties. Soil Science Society of American. Journal, v.47, p.770-775, 1983.

RITCHIE, J.T. Soil water availability. Plant Soil, v.58, p.327-338, 1981.

RODER, W.; MASON, S.C.; CLEGG, M.D.; KNIEFP, K.R. Yield-soil relationships in sorghum-soybean cropping systems with different fertilizer regimes. Agronomy Journal, v.81, n.3, p.470-475, 1989.

SLIFE, F.W. Economics of herbicide use and cultivar tolerance to herbicides. In: ANNUAL CORN SORGHUM RESEARCH CONFERENCE, 31 ., 1976, Chicago. Proceedings... Washington: American Seed Trade Association, 1976. p.77-82.

TANNER, C.B.; SINCLAIR, T.R. Efficient water use in crop production. In: TAYLOR, H.M.; JORDAN, W.R.; SINCLAIR, T.R. (Eds.). Limitation to efficient water use in crop production. Madison: American Society of Agronomy, 1983. p.1-27.

WAGGER, M.G. Timing effects of cover crop desiccation on decomposition rates and subsequent nitrogen uptake by corn. In: POWER, J.F. (Ed.). The role of legumes in conservation tillage systems: the proceedings of a national conference. Ankeny: Soil Conservation Society of America, 1987. p.35-37.

WAGGER, M.G.; MENGEL, D.B. The role of nonleguminous cover crops in the efficient use os water and nitrogen. In: HARGROVE, W.L. (Ed.). Cropping strategies for efficient use of water and nitrogen. Madison: Agronomy Society of American, 1988. p.41-59. (ASA. Special Publication, 51).

WILLIAMS, L.E.; SCHMITTHENNER, A.F. Effect of crop rotation on soil fungus populations. Phytopathology, v.52, p.241-247, 1962. 